\title{
Lower Bounds on Solutions of Quadratic Polynomials Defined over Finite Rings
}

\begin{abstract}
Ali H. Hakami
Department of Mathematics, Faculty of Science, Jazan University, P.O. Box 277, Jazan 45142, Saudi Arabia

Correspondence should be addressed to Ali H. Hakami; aalhakami@jazanu.edu.sa

Received 17 January 2015; Revised 16 April 2015; Accepted 20 April 2015

Academic Editor: Harvinder S. Sidhu

Copyright (c) 2015 Ali H. Hakami. This is an open access article distributed under the Creative Commons Attribution License, which permits unrestricted use, distribution, and reproduction in any medium, provided the original work is properly cited.

Let $m$ be a positive integer and let $R_{m}$ denote the ring $\mathbb{Z} /(m)$, and let $R_{m}^{n}$ denote the Cartesian product of $n$ copies of $\mathbb{Z} / m$. Let $f(\mathbf{x})$ be a quadratic polynomial in $\mathbb{Z}\left[x_{1}, \ldots, x_{n}\right]$. In this paper, we are interested in giving lower bounds on the number of solutions of the quadratic polynomial $f$ over the ring $R_{m}^{n}$.
\end{abstract}

\section{Introduction}

Let $R_{m}=\mathbb{Z} /(m)$ be a finite ring with $m$ positive integer. Let $f(\mathbf{x})$ be a quadratic polynomial in $\mathbb{Z}\left[x_{1}, \ldots, x_{n}\right]$. Write

$$
f(\mathbf{x})=Q(\mathbf{x})+\mathbf{a} \cdot \mathbf{x}+c,
$$

where $\mathbf{a} \in \mathbb{Z}^{n}, c \in \mathbb{Z}$, and $Q(\mathbf{x})$ is a quadratic form given by

$$
Q(\mathbf{x})=\frac{1}{2} \mathbf{x} A \mathbf{x}^{T},
$$

where $A$ is a symmetric $n \times n$ matrix with integer entries. Throughout this paper (with the exception of Lemma 4), we will assume that $\operatorname{gcd}(\operatorname{det} A, m)=1$. Let $V$ be the algebraic subset of $R_{m}^{n}$ defined by

$$
f(\mathbf{x}) \equiv 0 \quad(\bmod m) .
$$

If $d \mid m$ and $\mathbf{x}, \mathbf{y} \in R_{m}^{n}$, we will say that $\mathbf{x} \equiv \mathbf{y}(\bmod d)$ if $\mathbf{x}$ is congruent to $\mathrm{y}$ modulo the ideal $d R_{m}$. For any subset $S$ of $R_{m}^{n}$ and divisor $d$ of $m$, let

$$
\eta(S, d)=\left|\left\{\left(s_{1}, s_{2}\right) \in S \times S: s_{1} \equiv s_{2}(\bmod d)\right\}\right| .
$$

Let $\phi$ denote the Euler phi-function, let $\tau(m)$ denote the number of distinct positive divisors of $m$, and for positive integers $m$ and $n$ set

$$
\Phi_{n}(m)=\sum_{\substack{d>1 \\ d \mid m}} \phi(d) d^{-n / 2} .
$$

Considerable attention has been given to the problem of finding zeros of any polynomial over finite fields; see for example [1-8]. A special case of particular interest when the polynomial is quadratic over finite rings is studied in [9] by the author who obtained the following.

Theorem 1. For any subsets $S$ and $T$ of $R_{m}^{n}$, with $|S| \leq|T|$, we have

$$
\begin{aligned}
& |(S+T) \cap V| \\
& \geq m^{-1}|T| \\
& \quad-m^{-1}|S|^{-1} \sum_{\substack{d>m \\
d \mid m}} \phi(d) d^{n / 2} \eta(S, d)^{1 / 2} \eta(T, d)^{1 / 2} .
\end{aligned}
$$

In this paper, we will make the above result more precise when $S+T$ is a box of points in $R_{m}^{n}$, that is, the image $\overline{\mathscr{B}}$ of a box $\mathscr{B}$ in $\mathbb{Z}^{n}$ under the canonical mapping of $\mathbb{Z}^{n}$ onto $R_{m}^{n}$, where

$$
\overline{\mathscr{B}}=\left\{\mathbf{x} \in \mathbb{Z}^{n}: a_{i} \leq x_{i}<a_{i}+m_{i}\right\},
$$

for some $a_{i}, m_{i} \in \mathbb{Z}$ with $0<m_{i} \leq m, 1 \leq i \leq n$. In this case we obtain the following.

Theorem 2. Suppose that $n \geq 4$. Let $\overline{\mathscr{B}}$ be a box in $R_{m}^{n}$ whose sides are all of the same length $M<m$; that is, let $\overline{\mathscr{B}}$ be the 
image of a box $\mathscr{B}$ as given in (7), where $m_{i}=M, 1 \leq i \leq n$. Put $c=[(M+1) / 2]$. Then,

$$
\begin{gathered}
|\overline{\mathscr{B}} \cap V| \geq \frac{c^{n}}{m}\left(1-\Phi_{n}(m)-2^{n} \frac{\tau(m)}{c}\right. \\
\left.-\frac{m^{(m / 2)+1}}{c^{n}}\left(2^{n} \frac{c}{m}+1\right)\right) .
\end{gathered}
$$

In particular, if $n \geq 6$ and $M \gg m^{1 / 2+1 / n}$, then $\overline{\mathscr{B}} \cap V$ is nonempty.

The second part of Theorem 2 follows immediately from Lemma 3 of the next section. The first part of the theorem will be proven in Section 3.

\section{Auxiliary Lemmas}

Lemma 3. If $n \geq 4$, then for any integer $m>1$,

$$
\Phi_{n}(m) \leq \prod_{p \mid m}\left(1+p^{1-(n / 2)}\right)-1,
$$

where the product is over all primes $p$ dividing $m$. In particular, if $n \geq 6$, then for all $m>1, \Phi_{n}(m) \leq 2^{2-(n / 2)}$.

Proof. Let $s=n / 2$ and set $g(m)=\sum_{d \mid m} \phi(d) d^{-s}$, so that $\Phi_{n}(m)=g(m)-1$. Since $\phi(d)$ and $d^{-s}$ are both multiplicative, $g(m)$ is multiplicative. If $p$ is a prime and $e \geq 1$, then

$$
\begin{aligned}
g\left(p^{e}\right) & =1+\sum_{i=1}^{e}\left(p^{i}-p^{i-1}\right) p^{-s i} \\
& =1+\left(1-p^{-1}\right) \sum_{i=1}^{e} p^{(1-s) i} \\
& <1+\left(1-p^{-1}\right) p^{1-s} \sum_{i=0}^{\infty} p^{(1-s) i} \\
& =1+p^{1-s} \frac{1-p^{-1}}{1-p^{1-s}} \leq 1+p^{1-s}
\end{aligned}
$$

The last inequality follows since $s \geq 2$. The first part of the lemma now follows from the multiplicative property of $g(m)$. that

Now suppose that $n \geq 6$. Again, letting $s=n / 2$ we can say

$$
\Phi_{n}(m) \leq \sum_{d=2}^{\infty} \phi(d) d^{-s} \leq \frac{1}{2^{s}}+\frac{2}{3^{s}}+\frac{2}{4^{s}}+\sum_{d=5}^{\infty} d^{1-s},
$$

since $\phi(d)<d$. But

$$
\sum_{d=5}^{\infty} d^{1-s}<\int_{4}^{\infty} x^{1-s} d x=(s-2)^{-1} 4^{2-s} .
$$

Thus,

$$
\begin{aligned}
\Phi_{n}(m) & <\frac{1}{2^{s}}\left(1+2\left(\frac{2}{3}\right)^{s}+\frac{1}{2^{s-1}}+\frac{1}{2^{s-4}(s-2)}\right) \\
& \leq \frac{1}{2^{s}} \cdot 4, \quad \text { for } s \geq 3 .
\end{aligned}
$$

To prove Theorem 2, we make use of exponential sums. Let $e_{m}(x)=e^{(2 \pi i / m) x}$. We will abbreviate complete sums $\sum_{x \in R_{m}^{n}}()$ by simply $\sum_{x}()$. Also we will need to use the following fundamental identity: for any $\mathbf{y} \in R_{m}^{n}$,

$$
\sum_{x} e_{m}(\mathbf{x} \cdot \mathbf{y})= \begin{cases}m^{n} & \text { if } \mathbf{y}=\mathbf{0} \\ 0 & \text { if } \mathbf{y} \neq \mathbf{0} .\end{cases}
$$

Let $f(\mathbf{x})$ and $Q(\mathbf{x})$ be as defined by (1) and (2). By viewing $R_{m}^{n}$ as a $\mathbb{Z}$-module, the Gauss sums

$$
\begin{aligned}
& G_{m}(Q, \mathbf{y})=\sum_{x \in R_{m}^{n}} e_{m}(Q(\mathbf{x})+\mathbf{y} \cdot \mathbf{x}) \\
& G_{m}(f, \mathbf{y})=\sum_{x \in R_{m}^{n}} e_{m}(f(\mathbf{x})+\mathbf{y} \cdot \mathbf{x})
\end{aligned}
$$

are well defined whether we take $\mathbf{y} \in \mathbb{Z}^{n}$ or $\mathbf{y} \in R_{m}^{n}$.

For any $n \times n$ matrix $A$ with integer entries, we define $\operatorname{ker}_{m}(A)$ by

$$
\operatorname{ker}_{m}(A)=\left\{\mathbf{x} \in R_{m}^{n}: A \mathbf{x}^{T} \equiv \mathbf{0}^{T}(\bmod m)\right\} .
$$

We need the following lemmas.

Lemma 4 (see [10, Theorem 4]). Let $Q(\mathbf{x})$ be a quadratic form given by (2), where now we allow A to be any symmetric integral matrix with even diagonal entries. Then, given $\mathbf{y} \in R_{m}^{n}$, the Gauss sum $G_{m}(Q, \mathbf{y})$ is zero unless $Q(\mathbf{x})+\mathbf{y} \cdot \mathbf{x} \equiv 0(\bmod m)$ for all $\mathbf{x} \in \operatorname{ker}_{m}(A)$, in which case $\left|G_{m}(Q, \mathbf{y})\right|^{2}=m^{n}\left|\operatorname{ker}_{m} A\right|$.

Lemma 5. Let $f(\mathbf{x})$ be a quadratic polynomial as given by (1) with $\operatorname{gcd}(\operatorname{det} A, m)=1$. Let $\mathbf{y} \in \mathbb{Z}^{n}, \lambda \in \mathbb{Z}, \lambda \neq \equiv 0(\bmod m)$, and set $d=(\lambda, m)$. Then,

$$
\left|G_{m}(\lambda f, \mathbf{y})\right|= \begin{cases}(m d)^{n / 2} & \text { if } \mathbf{y} \equiv \mathbf{0}(\bmod d), \\ 0 & \text { if } \mathbf{y} \neq \equiv \mathbf{0}(\bmod d) .\end{cases}
$$

Proof. By (1), we have

$$
\begin{aligned}
\left|G_{m}(\lambda f, y)\right| & =\left|\sum_{\mathbf{x}} e_{m}(\lambda f(\mathbf{x})+\mathbf{y} \cdot \mathbf{x})\right| \\
& =\left|\sum_{\mathbf{x}} e_{m}(\lambda Q(\mathbf{x})+(\lambda \mathbf{a}+\mathbf{y}) \cdot \mathbf{x}+\lambda c)\right| \\
& =\left|\sum_{\mathbf{x}} e_{m}(\lambda Q(\mathbf{x})+(\lambda \mathbf{a}+\mathbf{y}) \cdot \mathbf{x})\right| .
\end{aligned}
$$

Now $\lambda Q(\mathbf{x})=(1 / 2) \mathbf{x}(\lambda A) \mathbf{x}^{T}$, so that by Lemma $4, G_{m}(\lambda f$, $\mathbf{y})=0$ unless $\mathbf{y}$ satisfies the following condition:

$$
\forall \mathbf{x} \in \operatorname{ker}_{m}(\lambda A),
$$

$$
(\lambda \mathbf{a}+\mathbf{y}) \cdot \mathbf{x}+\lambda Q(\mathbf{x}) \equiv 0(\bmod m)
$$

Now

$$
\begin{aligned}
\operatorname{ker}_{m}(\lambda A) & =\left\{\mathbf{x} \in R_{m}^{n}: \lambda A \mathbf{x}^{T} \equiv \mathbf{0}(\bmod m)\right\} \\
& =\left\{\mathbf{x} \in R_{m}^{n}: A \mathbf{x}^{T} \equiv \mathbf{0}\left(\bmod \frac{m}{d}\right)\right\},
\end{aligned}
$$


but as $\operatorname{gcd}(\operatorname{det} A, m)=1$ we conclude that

$$
\operatorname{ker}_{m}(\lambda A)=\left\{\mathbf{x} \in R_{m}^{n}: \mathbf{x} \equiv \mathbf{0}\left(\bmod \frac{m}{d}\right)\right\} .
$$

Thus, setting $\mathbf{x} \equiv(m / d) \mathbf{t}(\bmod m)$, we see that $(19)$ is equivalent to saying that, for all $\mathbf{t} \in \mathbb{Z}^{n}$,

$$
\begin{aligned}
& \lambda\left(\frac{m}{d}\right) \mathbf{a} \cdot \mathbf{t}+\left(\frac{m}{d}\right) \mathbf{y} \cdot \mathbf{t}+\lambda\left(\frac{m}{d}\right)^{2} Q(\mathbf{t}) \\
& \quad \equiv 0(\bmod m) .
\end{aligned}
$$

But $\lambda(m / d)=m(\lambda / d) \equiv 0(\bmod m)$ and similarly $\lambda(m / d)^{2}=$ $m(\lambda / d)(m / d) \equiv 0(\bmod m)$ so that $(22)$ simplifies to the congruence $(m / d) \mathbf{y} \cdot \mathbf{t} \equiv 0(\bmod m)$; that is, $\mathbf{y} \cdot \mathbf{t} \equiv 0(\bmod d)$. Hence, $\mathbf{y}$ satisfies (19) if and only if, for all $\mathbf{t} \in \mathbb{Z}^{n}, \mathbf{y} \cdot \mathbf{t} \equiv$ $0(\bmod d)$, that is $\mathbf{y} \equiv \mathbf{0}(\bmod d)$. If $\mathbf{y} \equiv \mathbf{0}(\bmod d)$ then by Lemma 4, and (21), we have

$$
\left|G_{m}(\lambda f, \mathbf{y})\right|^{2}=m^{2}\left|\operatorname{ker}_{m}(\lambda A)\right|=m^{n} d^{n} .
$$

\section{Proof of Theorem 2}

Let $S$ and $T$ be subsets of $R_{m}^{n}$, and let $V$ be the set of points in $R_{m}^{n}$ satisfying $f(\mathbf{x}) \equiv 0(\bmod m)$. Let $N$ be the number of triples $(\mathbf{s}, \mathbf{t}, \mathbf{v}) \in S \times T \times V$ such that $\mathbf{s}+\mathbf{t} \equiv \mathbf{v}(\bmod m)$.

Lemma 6. Let $N$ be the number of triples $(\mathbf{s}, \mathbf{t}, \mathbf{v}) \in S \times T \times V$ such that $\mathbf{s}+\mathbf{t} \equiv \mathbf{v}(\bmod m)$. Then, for any subsets $S$ and $T$ of $R_{m}^{n}$, with $|S| \leqslant|T|$ we have

$$
\begin{aligned}
& \left|N-m^{-1}\right| S|| T|| \\
& \quad \leq m^{-1} \sum_{\substack{d>1 \\
d \mid m}} \phi(d) d^{-n / 2} d^{n} \eta(S, d)^{1 / 2} \eta(T, d)^{1 / 2} .
\end{aligned}
$$

Proof. By the fundamental identity (14),

$$
\begin{aligned}
N & =m^{-n} \sum_{\mathbf{x} \in V} \sum_{\mathbf{s} \in S} \sum_{\mathbf{t} \in T} \sum_{\mathbf{y}} e_{m}(\mathbf{y} \cdot(\mathbf{s}+\mathbf{t}-\mathbf{x})) \\
& =m^{-n-1} \sum_{\mathbf{x} \in R_{m}^{n}}\left[\sum_{\lambda \in R_{m}} e_{m}(\lambda f(\mathbf{x}))\right] \\
& \cdot \sum_{\mathbf{s} \in S} \sum_{\mathbf{t} \in T} \sum_{\mathbf{y}} e_{m}(\mathbf{y} \cdot(\mathbf{s}+\mathbf{t}-\mathbf{x})) \\
& =m^{-n-1} \sum_{\lambda} \sum_{\mathbf{y}} \Psi(\mathbf{y}) \sum_{\mathbf{x}} e_{m}(\lambda f(\mathbf{x})-\mathbf{x}),
\end{aligned}
$$

where

$$
\Psi(\mathbf{y})=\sum_{\mathbf{s} \in S} \sum_{\mathbf{t} \in T} e_{m}(\mathbf{y} \cdot(\mathbf{s}+\mathbf{t}))
$$

Peeling off the $\lambda=0$ term yields

$$
\begin{aligned}
N= & m^{-1}|S| \cdot|T| \\
& +m^{-n-1} \sum_{\lambda \neq 0} \sum_{\mathbf{y}} \Psi(\mathbf{y}) \sum_{\mathbf{x}} e_{m}(\lambda f(\mathbf{x})-\mathbf{y} \cdot \mathbf{x}) .
\end{aligned}
$$

Thus (from (27)),

$$
\begin{aligned}
N & -m^{-1}|S| \cdot|T| \\
& =m^{-n-1} \sum_{\substack{1 \leq d<m \\
d \mid m}} \sum_{\substack{1 \leq \lambda<m \\
(\lambda, m)=d}} \sum_{\mathbf{y}} \Psi(\mathbf{y}) \sum_{\mathbf{x}} e_{m}(\lambda f(\mathbf{x})-\mathbf{y} \cdot \mathbf{x}),
\end{aligned}
$$

so that by Lemma 5 ,

$$
\begin{aligned}
& \left|N-m^{-1}\right| S|| T|| \\
& \quad \leq m^{-n-1} \sum_{\substack{d \mid m \\
d<m}} \sum_{\substack{1 \leq \lambda<m \\
(\lambda, m)=d}} m^{n / 2} d^{n / 2} \sum_{\mathbf{y}}^{*}|\Psi(\mathbf{y})|,
\end{aligned}
$$

where the sum $\sum^{*}$ on $\mathbf{y}$ is over all $\mathbf{y} \equiv \mathbf{0}(\bmod d)$. On replacing $d$ by $m / d$ and $\sum_{\mathbf{y}}^{*}$ by $\sum_{\mathbf{y}}^{* *}$, the sum over all $\mathbf{y} \equiv$ $\mathbf{0}(\bmod (m / d))$, we obtain

$$
\begin{aligned}
& \left|N-m^{-1}\right| S|| T|| \\
& \leq m^{-n / 2-1} \sum_{\substack{d \mid m \\
d<m}} \phi\left(\frac{m}{d}\right) d^{n / 2} \sum_{\mathbf{y}}^{*}|\Psi(\mathbf{y})| \\
& =m^{-1} \sum_{\substack{d>1 \\
d \mid m}} \phi(d) d^{-n / 2} \sum_{\mathbf{y}}^{* *}|\Psi(\mathbf{y})|
\end{aligned}
$$

Now,

$$
\begin{aligned}
\sum_{\mathbf{y}}^{* *} & |\Psi(\mathbf{y})||\Psi(\mathbf{y})|=\sum_{\mathbf{y}}^{* *}\left|\sum_{\mathbf{s} \in S} e_{m}(\mathbf{y} \cdot \mathbf{s})\right|\left|\sum_{\mathbf{t} \in T} e_{m}(\mathbf{y} \cdot \mathbf{t})\right| \\
\leq & {\left[\sum_{\mathbf{y}}^{* *}\left|\sum_{\mathbf{s} \in S} e_{m}(\mathbf{y} \cdot \mathbf{s})\right|^{2}\right]^{1 / 2} } \\
\cdot & {\left[\sum_{\mathbf{y}}^{* *}\left|\sum_{\mathbf{t} \in T} e_{m}(\mathbf{y} \cdot \mathbf{t})\right|^{2}\right]^{1 / 2} . }
\end{aligned}
$$

Setting $\mathbf{y} \equiv(m / d) \mathbf{u}(\bmod m)$ and letting $\mathbf{u}$ run through a complete set of representatives for $R_{d}^{n}$, we can say

$$
\begin{aligned}
\sum_{\mathbf{y}}^{* *}\left|\sum_{\mathbf{t} \in T} e_{m}(\mathbf{y} \cdot \mathbf{t})\right|^{2} \\
=\sum_{\mathbf{y}}^{* *} \sum_{\mathbf{s}_{1} \in S} \sum_{\mathbf{s}_{2} \in S} e_{m}\left(\mathbf{y} \cdot\left(\mathbf{s}_{1}-\mathbf{s}_{2}\right)\right) \\
=\sum_{\mathbf{s}_{1} \in S} \sum_{\mathbf{s}_{2} \in S} \sum_{\mathbf{y}}^{* *} e_{m}\left(\mathbf{y} \cdot\left(\mathbf{s}_{1}-\mathbf{s}_{2}\right)\right) \\
=\sum_{\mathbf{s}_{1} \in S} \sum_{\mathbf{s}_{2} \in S} \sum_{\mathbf{u} \in R_{d}^{n}} e_{m}\left(\frac{m}{d} \mathbf{u} \cdot\left(\mathbf{s}_{1}-\mathbf{s}_{2}\right)\right) \\
=\sum_{\mathbf{s}_{1} \in S} \sum_{\mathbf{s}_{2} \in S} \sum_{\mathbf{u}} e_{d}\left(\mathbf{u} \cdot\left(\mathbf{s}_{1}-\mathbf{s}_{2}\right)\right)=d^{n} \eta(S, d) .
\end{aligned}
$$

Thus, by (31),

$$
\sum_{\mathbf{y}}^{* *}|\Psi(\mathbf{y})| \leq d^{n} \eta(S, d)^{1 / 2} \eta(T, d)^{1 / 2}
$$


and therefore, by (30),

$$
\begin{aligned}
& \left|N-m^{-1}\right| S|| T|| \\
& \quad \leq m^{-1} \sum_{\substack{d>1 \\
d \mid m}} \phi(d) d^{-n / 2} d^{n} \eta(S, d)^{1 / 2} \eta(T, d)^{1 / 2} .
\end{aligned}
$$

Proof of Theorem 2. Let

$$
\begin{aligned}
& B=\left\{\mathbf{x} \in \mathbb{Z}^{n}: a_{i} \leq x_{i}<a_{i}+M, 1 \leq i \leq n\right\}, \\
& S=\left\{\mathbf{x} \in \mathbb{Z}^{n}: 0 \leq x_{i}<c\right\}, \\
& T=S+\left(a_{1}, a_{2}, \ldots, a_{n}\right),
\end{aligned}
$$

for some $a_{i} \in \mathbb{Z}, 1 \leq i \leq n$, where $c=[(M+1) / c]$. Let $\bar{B}, \bar{S}$, and $\bar{T}$ be the images of $B, S$, and $T$ in $R_{m}^{n}$ under the canonical mapping of $\mathbb{Z}^{n}$ onto $R_{m}^{n}$. Then, $\bar{S}+\bar{T} \subset \bar{B}$ and $|\bar{S}|=|\bar{T}|=$ $[(M+1) / c]^{n} \geq 2^{-n}|\bar{B}|$. We claim that for any divisor $d$ of $m$,

$$
\eta(\bar{S}, d) \leq c^{n}\left(\left[\frac{c}{d}\right]+1\right)^{n}
$$

and that the same inequality holds for $\eta(\bar{T}, d)$. Let $\mathbf{s}=$ $\left(s_{1}, \ldots, s_{n}\right)$ be a fixed point in $S$. If $\mathbf{u}=\left(u_{1}, \ldots, u_{n}\right)$ is a point in $S$ such that $\mathbf{u} \equiv \mathbf{s}(\bmod d)$, then $u_{i}=s_{i}+d k_{i}$, for some $k_{i} \in \mathbb{Z}, 1 \leq i \leq n$. Since $0 \leq u_{i}<c$, there are at most $[c / d]+1$ choices for each $u_{i}$, and thus at most $([c / d]+1)^{n}$ choices for $\mathbf{u}$. Since $|S|=c^{n}$, we obtain (36). It is clear that $\eta(\bar{S}, d)=\eta(\bar{T}, d)$ so that (36) holds also for $\eta(\bar{T}, d)$.

We now apply Theorem 1 with $S$ and $T$ replaced by the sets $\bar{S}$ and $\bar{T}$ just defined. We will abbreviate the sum $\sum_{d>1, d \mid m}$ by simple $\sum^{*}$. From Lemma 6 and (36) we have

$$
\begin{aligned}
\left|N-m^{-1}\right| S|| T|| & \leq m^{-1} \sum^{*} \phi(d) d^{n / 2} c^{n}\left(\left[\frac{c}{d}\right]+1\right)^{n} \\
& \leq m^{-1} \sum^{*} \phi(d) d^{n / 2} c^{n} d^{-n}(c+d)^{n} \\
& =m^{-1} c^{n} \sum^{*} \phi(d) d^{-n / 2} c^{n}(c+d)^{n} .
\end{aligned}
$$

Now, since $(c+d)^{n} \leq c^{n}+2^{n}\left(c^{n-1} d+c d^{n-1}\right)+d^{n}$, we have

$$
\begin{aligned}
\left|N-m^{-1}\right| S|| T|| \leq & m^{-1} c^{2 n} \sum^{*} \phi(d) d^{-n / 2} \\
& +m^{-1} 2^{n} c^{2 n-1} \sum^{*} \phi(d) d^{1-n / 2} \\
& +m^{-1} 2^{n} c^{n+1} \sum^{*} \phi(d) d^{(n / 2)-1} \\
& +m^{-1} c^{n} \sum^{*} \phi(d) d^{n / 2} .
\end{aligned}
$$

The first sum on the right-hand side of (38) is just $\Phi_{n}(m)$. We make crude estimates for the remaining sums. For $n \geq 4$ we have

$$
\begin{aligned}
\sum^{*} \phi(d) d^{1-(n / 2)} & \leq \sum^{*} \phi(d) d^{-1} \leq \sum^{*} 1<\tau(m), \\
\sum^{*} \phi(d) d^{(n / 2)-1} & \leq m^{n / 2-1} \sum^{*} \phi(d) \leq m^{n / 2-1} \cdot m \\
& =m^{n / 2} \\
\sum^{*} \phi(d) d^{(n / 2)} & \leq m^{n / 2} \sum^{*} \phi(d) \leq m^{n / 2+1} .
\end{aligned}
$$

Thus, by (38), we see that

$$
\begin{aligned}
N & \geq m^{-1} c^{2 n}-m^{-1} c^{2 n} \Phi_{n}(m)-m^{-1} 2^{n} c^{2 n-1} \tau(m) \\
& -m^{-1} 2^{n} c^{n+1} m^{n / 2}-m^{-1} c^{n} m^{(n / 2)+1}=m^{-1} c^{2 n}(1 \\
& -\Phi_{n}(m)-2^{n} c^{-1} \tau(m) \\
& \left.-c^{-n} m^{(n / 2)+1}\left(2^{n} c m^{-1}+1\right)\right) .
\end{aligned}
$$

Theorem 2 now follows from the observation that

$$
|\bar{B} \cap V| \geq|(\bar{S} \cap \bar{T}) \cap V| \geq N|S|^{-1}=N c^{-n} .
$$

\section{Remarks}

(1) It is clear from (39) and Lemma 3 that if $n \geq 8$ then in the statement of Theorem 2 we can replace $\tau(m)$ by $2^{3-(n / 2)}$

(2) Let $f(\mathbf{x})$ be given by (1), let $V$ be the set of zeros of $f(\mathbf{x})$ in $R_{m}^{n}$, and again suppose that $\operatorname{gcd}(\operatorname{det} A, m)=$ 1. Lemma 5 provides us with an easy means of estimating $|V|$. For $n \geq 4$, we obtain

$$
|V|=m^{n-1} \prod_{p \mid m}\left(1+\theta_{p} p^{1-(n / 2)}\right)
$$

where the product is over all primes $p$ dividing $m$, and for each such $p, \theta_{p}$ is a real number of absolute value $\leq 1$. Equation (42) follows from the observation that

$$
\begin{aligned}
|V| & =m^{-1} \sum_{\mathbf{x} \in R_{m}^{n}} \sum_{\lambda=0}^{m-1} e(\lambda f(\mathbf{x})) \\
& =m^{n-1}+m^{-1} \sum_{\lambda=1}^{m-1} \sum_{\mathbf{x}} e(\lambda f(\mathbf{x})) .
\end{aligned}
$$

By Lemma 5, we then have

$$
|V|=m^{n-1}+\theta_{m} m^{n / 2-1} \sum_{\substack{1 \leq d<m \\ d \mid m}} \phi\left(\frac{m}{d}\right) d^{n / 2},
$$


for some $\theta_{m} \in \mathbb{R}$, with $\left|\theta_{m}\right| \leq 1$. Since

$$
\Phi_{n}(m)=\sum_{\substack{d>1 \\ d \mid m}} \phi(d) d^{-n / 2}=\sum_{\substack{1 \leq d<m \\ d \mid m}} \phi\left(\frac{m}{d}\right)\left(\frac{d}{m}\right)^{n / 2}
$$

we obtain

$$
|V|=m^{n-1}\left[1+\theta_{m} \Phi_{n}(m)\right] .
$$

To obtain (42), we apply (46) in turn to each prime power dividing $m$ and use the Chinese remainder theorem to compute $|V|$. That is, for each divisor $d$ of $m$, we let $v(d)$ be the number of points in $R_{d}^{n}$ satisfying the congruence $f(\mathbf{x}) \equiv 0(\bmod d)$. Consequently, if $m=\prod_{i=1}^{s} p_{i}^{e_{i}}$ then $v(m)=$ $\prod_{i=1}^{s} v\left(p_{i}^{e_{i}}\right)$. Thus, by (46), we have

$$
|V|=v(m)=\prod_{i=1}^{s} p_{i}^{e_{i}(n-1)}\left(1+\theta_{i} \Phi_{n}\left(p_{i}^{e_{i}}\right)\right),
$$

for some $\theta_{i} \in \mathbb{R}$ with $\left|\theta_{i}\right| \leq 1,1 \leq i \leq s$, and by Lemma 3 we have

$$
\Phi_{n}\left(p^{e}\right) \leq p^{1-(n / 2)},
$$

for any prime power $p^{e}$, when $n \geq 4$. Equation (42) is now immediate.

Equation (42) indicates that we obtain roughly the expected quota of zeros for $f(\mathbf{x})$, namely, $m^{n-1}$, when $\operatorname{gcd}(\operatorname{det} A, m)=1$. When $\operatorname{gcd}(\operatorname{det} A, m) \neq 1$, this is no longer the case. For example, suppose that $m=p q$, where $p$ and $q$ are distinct primes. Let $\alpha$ be quadratic nonresidue $(\bmod p)$, let $\beta$ be a quadratic nonresidue $(\bmod q)$, and let $f(\mathbf{x})=f\left(x_{1}, x_{2}, x_{3}, x_{4}\right)$ be defined by

$$
f(\mathbf{x})=p\left(x_{1}^{2}-\beta x_{2}^{2}\right)+q\left(x_{3}^{2}-\alpha x_{4}^{2}\right) .
$$

If $\mathbf{x}$ is an integral solution of the congruence $f(\mathbf{x}) \equiv$ $0(\bmod m)$ then $x_{1}^{2}-\beta x_{2}^{2} \equiv 0(\bmod q)$ and $x_{3}^{2}-\alpha x_{4}^{2} \equiv$ $0(\bmod p)$, so that $x_{1} \equiv x_{2} \equiv 0(\bmod q)$ and $x_{3} \equiv x_{4} \equiv$ $0(\bmod p)$. Thus, if $V$ is the set of points in $R_{m}^{4}$ satisfying $f(\mathbf{x}) \equiv 0(\bmod m)$, then $|V|=p^{2} q^{2}=m^{2}$, rather than expected quota of $m^{3}$. This example indicates that Theorem 2 does not hold when $\operatorname{gcd}(\operatorname{det} A, m) \neq 1$.

We have not been able to obtain an analogue of Theorem 2 when $\operatorname{gcd}(\operatorname{det} A, m) \neq 1$. The main difficulty is that $\operatorname{ker}_{m}(\lambda A)$ no longer leads to such a simple description as in the case when $\operatorname{gcd}(\operatorname{det} A, m)=1$; see (21). To overcome this difficulty, one may be able to use the description of $\operatorname{ker}_{m}(\lambda A)$ given in section 4 of [11] which involved the invariant factors of $A$.

\section{Conflict of Interests}

The author has no competing interests.

\section{Acknowledgments}

The author is very grateful to the referees and the editors for their valuable comments and some helpful suggestions that improved the clarity and readability of the paper. The author is thankful to Jazan University (JU), Jazan, Saudi Arabia, for providing excellent research facilities to carry out this research.

\section{References}

[1] T. Cochrane, "The distribution of solutions to equations over finite fields," Transactions of the American Mathematical Society, vol. 293, no. 2, pp. 819-826, 1986.

[2] L. J. Mordell, "On the number of solutions in incomplete residue sets of quadratic congruences," Archiv der Mathematik, vol. 8, pp. 153-157, 1957.

[3] L. J. Mordell, "Incomplete exponential sums and incomplete residue systems for congruences," Czechoslovak Mathematical Journal, vol. 14 (89), pp. 235-242, 1964.

[4] J. H. H. Chalk, "The number of solutions of congruences in incomplete residue system," Canadian Journal of Mathematics, vol. 15, pp. 291-296, 1963.

[5] J. H. H. Chalk and K. S. Williams, "The distribution of solutions of congruences," Mathematika, vol. 12, pp. 176-192, 1965.

[6] G. Myerson, "The distribution of rational points on varieties defined over a finite field," Mathematika, vol. 28, no. 2, pp. 153159, 1981.

[7] R. A. Smith, "The distribution of rational points on hypersurfaces defined over a finite field," Mathematika, vol. 17, pp. 328 332, 1970.

[8] K. W. Spackman, "On the number and distribution of simultaneous solutions to diagonal congruences," Canadian Journal of Mathematics, vol. 33, no. 2, pp. 421-436, 1981.

[9] A. H. Hakami, "The distribution of solutions to quadratic polynomials over finite rings," Journal of Mathematical Sciences. Advances and Applications, vol. 17, no. 1-2, pp. 39-52, 2012.

[10] J. H. Loxton and R. A. Smith, "Estimates for multiple exponential sums," Journal of the Australian Mathematical Society, Series A, vol. 33, no. 1, pp. 125-134, 1982.

[11] T. Cochrane, "Small solutions of congruences over algebraic number fields," Illinois Journal of Mathematics, vol. 31, no. 4, pp. 618-625, 1987. 


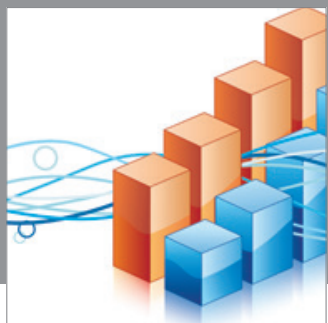

Advances in

Operations Research

mansans

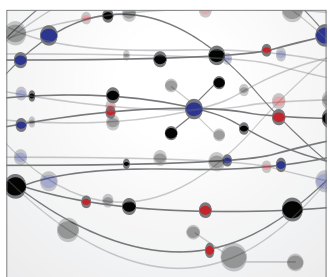

The Scientific World Journal
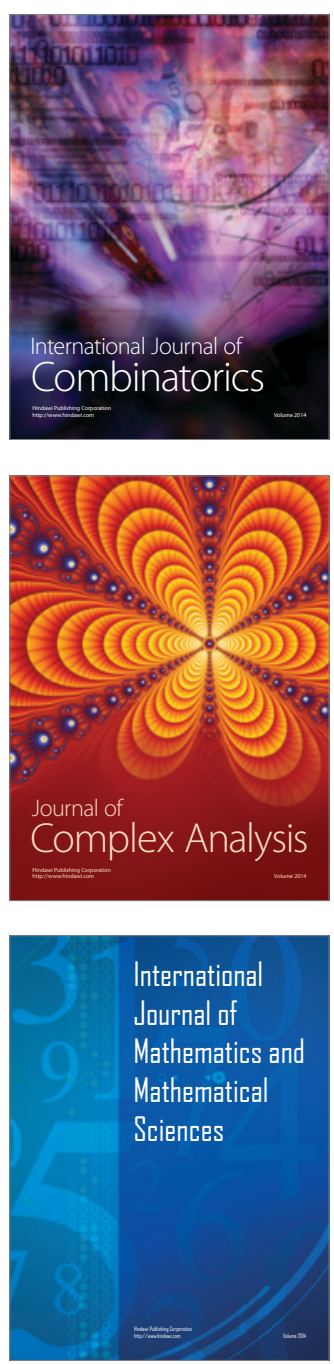
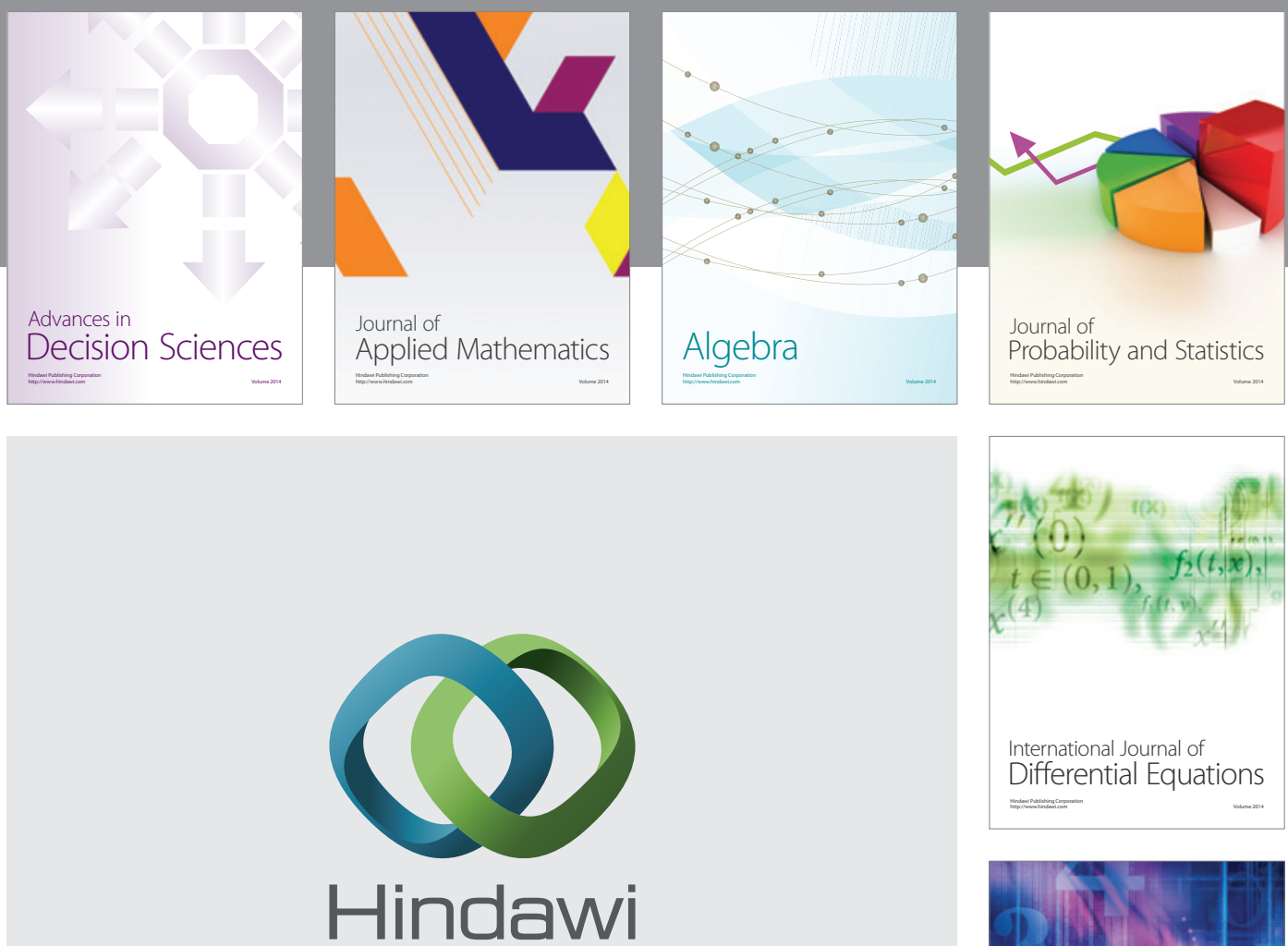

Submit your manuscripts at http://www.hindawi.com
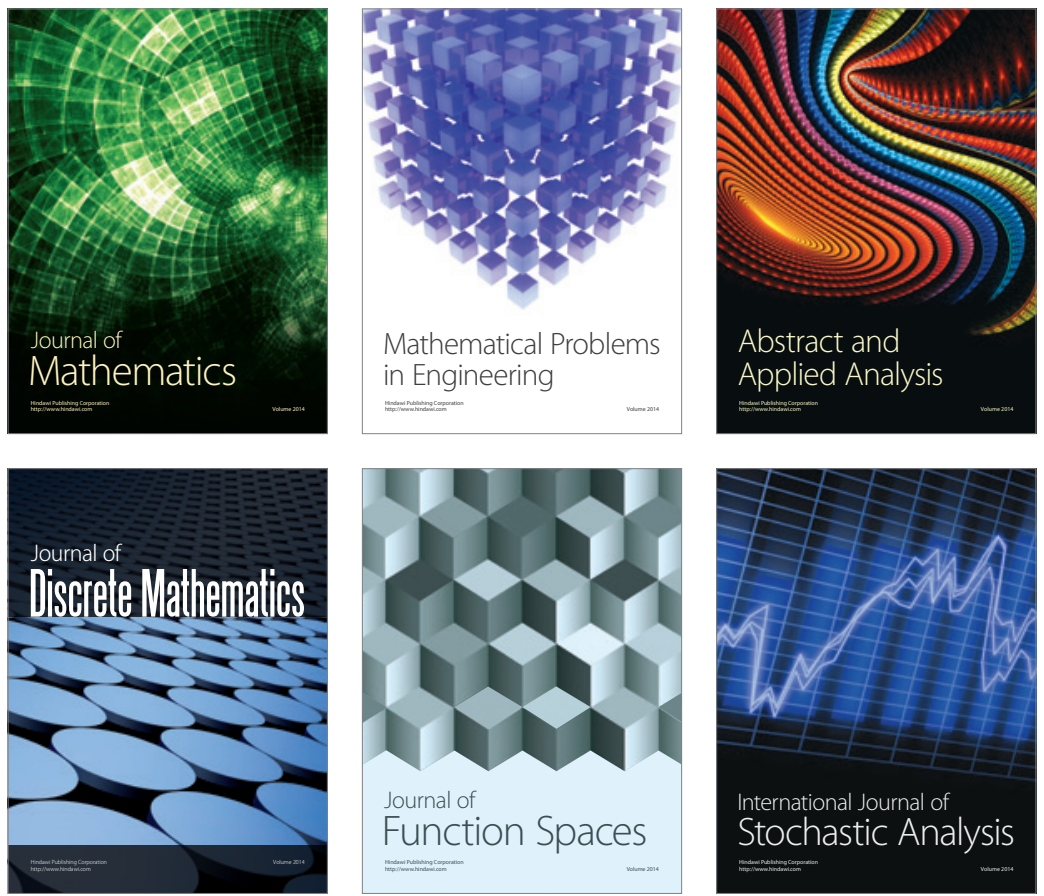

Journal of

Function Spaces

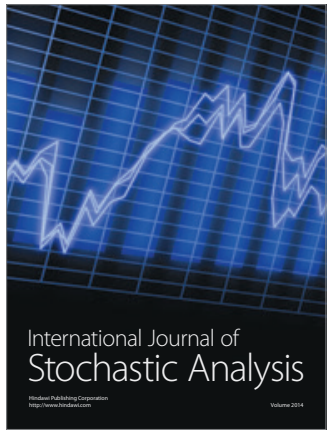

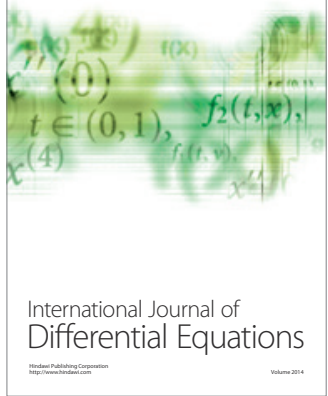
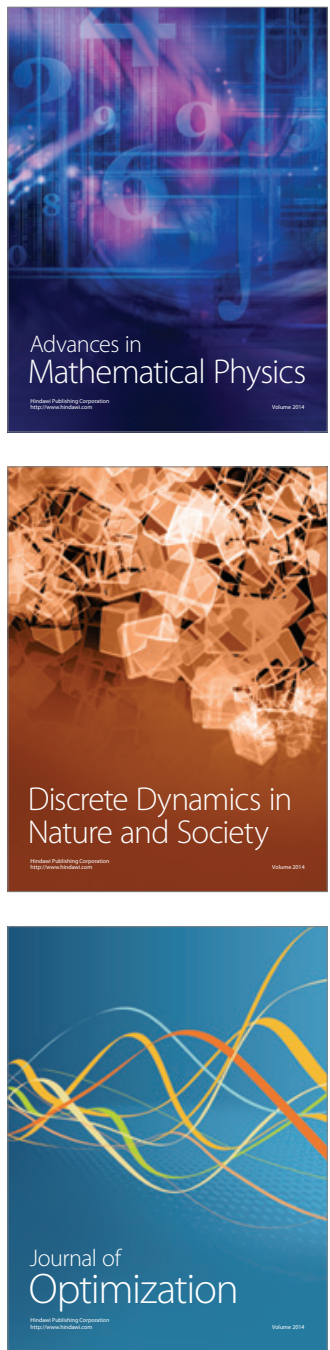